\title{
On the potential yield of wind turbines on high-rise buildings
}

\author{
Giulio Vita ${ }^{1}$, Simone Salvadori² ${ }^{*}$, Anina Sarkić-Glumac $^{3}$, Daniela Anna Misul ${ }^{2}$ \\ ${ }^{1}$ Dipartimento di Ingegneria Industriale (DIEF), Università degli Studi Firenze, Firenze, IT \\ ${ }^{2}$ Dipartimento Energia (DENERG), Politecnico di Torino, Torino, IT \\ ${ }^{3}$ Interdisciplinary Centre for Security, Reliability and Trust (SnT), University of Luxembourg, Luxembourg, LU
}

\begin{abstract}
Urban wind energy has intrigued some investments and applications over the last decade. However, most applications have been unsuccessful, in many cases leaving behind non-rotating wind turbines in the built environment. Research on urban wind energy has also lacked in providing a reliable description of the local flow features small wind turbines are placed in, and the positioning strategy is mostly based on empiricism. This study uses data from a wind tunnel test on the flow pattern above the roof of high-rise buildings to estimate the potential yield of small wind turbines installed in various configurations. The data are collected at a height of $12 \mathrm{~m}$ above the flat roof under various wind directions. The capacity factor of a medium-size horizontal axis wind turbine is calculated using power curve data available from the literature. The local wind resource is calculated using the mean wind speed as measured with hot-wire anemometry. The annual energy production of the various configurations is calculated using the climate data available for Firenze (IT) and the orientation of the building with respect to main winds. A rather large variability in the power output of the roof-farm was found for the chosen configuration.
\end{abstract}

\section{Introduction}

Urban Wind Energy is a niche of wind energy aiming at harvesting the wind resource in the built environment. Actually, available urban wind energy applications have been able to provide but a tiny fraction of the electricity promised [1]. It is no wonder that from a surge in research investigation and number of start-ups producing small wind turbines for installation in the built environment, a quite generalized opinion has taken place that urban wind energy is simply not worth the trouble and the money [2]. In fact, there are essentially two reasons for this debacle: the wind energy resource on buildings is an area of knowledge yet to be explored and the performance of wind turbines in highly turbulent environments is mostly unknown.

The flow pattern around buildings has received much attention over the past decades and so has the wind loading on buildings. More specifically, the ultimate scope of the researches was to retrieve force coefficients to be used as input in the structural design of low- and high-rise buildings. However, in order to study the wind energy resource a different setup would be needed (wind tunnel or numerical results using full-scale data). Still, such data are not available as it is difficult to justify the expenditure of a field test campaign to measure the wind resource on top of buildings basing on the current performance of urban wind energy applications $[1,3]$.

In order to position wind turbines, a supposition should be made on the role of turbulence intensity, the latter being rather harmful for the performance of devices $[2,3,4]$. The few investigations available on the positioning of wind turbines agree that the critical turbulence intensity would occur quite far from the roof, at a critical height as high as $10 \mathrm{~m}$. Practically, the latter implies that wind turbines should be installed on the top of a tall mast [5].

Among the studies reporting on the experimental velocity field around high-rise buildings, only very few focus on the roof region and even less are dedicated to wind energy, being the scope of the work mostly focused on wind loading on buildings [3]. In [1] the authors have shown that wind tunnel testing is reliable in reproducing the wind energy resource above high-rise buildings reporting on a realistic urban geometry compared with full-scale data. In [6] the authors have investigated in detail the velocity flow field due to the presence of adjacent buildings for wind energy harvesting. A complex flow pattern is found to occur, which is characterized by a strong variability with the wind direction or the configuration of the roof shape. Furthermore, in the same set of wind tunnel experiments, the results presented in [7] have shown that

\footnotetext{
* Corresponding author: simone.salvadori@polito.it
} 
the flow above the roof might be actually exploited to increase the energy harvested taking advantage of the local accelerations of the flow. In further work in the same dataset it is also shown that the turbulent behavior is only present in the vicinity of the roof, while in most locations across the roof flow region the velocity field has a high potential for wind energy harvesting [8].

The potential of urban wind energy has been indeed debated in some researches, focusing on the calculation of the predicted Annual Energy Production (AEP). In [9] the authors estimate the potential urban wind energy yields in the Greater London area, based on available annual wind speed values only. They conclude that it is suitable to place devices far from the city center, where the annual wind speed is high and the building height is lower. Results were obtained based on mesoscale considerations for the wind speed, roughness characteristics at the site and stability. In [10] the authors follow a similar route and propose a framework to estimate the potential of urban wind energy in the Netherlands, based on the data available for high-rise buildings as well as on the annual wind speed distributions in 14 major cities. This study is interesting as it proposes the installation of 12 vertical-axis wind turbines (VAWT) at the corners of high-rise buildings, taking into account mutual disturbances to the flow based on an optimal design of the VAWT so as to limit interfering effects and maximize performance [11]. However, both studies disregard the local features of the flow that might significantly affect performance. In particular, in the vicinity of buildings, turbulence intensity [12], skewed and yawed flow conditions [13] might affect performance, regardless of the wind speed at the installation position. Furthermore, virtually no work exists in the literature on the effect of the wind direction and how it affects the behavior of the flow around high-rise buildings.

Hence, an important gap in the research is detectable to drive future efforts on urban wind energy. Present study aims at determining the potential AEP associated to a roof-farm built in the city of Firenze (IT). Several configurations for the positioning of a typical small wind turbine on the roof of a model high-rise building are considered. Results are based on experimental data available for high-rise buildings, annual wind speed distribution and local features of the flow.

\section{Methodology}

The methodology of this study is developed in order to work out the potential power performance of a rooffarms from a set of experimental data. The outcome is based on the use of the following datasets:

- Flow pattern around high-rise building: the flow pattern consists of measurements of the fluctuating wind speed above the roof where the roof-farm is to be placed. Three heights are considered, namely: $z=3 \mathrm{~m}$, which is suitable for small wind turbines; $z=12-18 \mathrm{~m}$, which is suitable for larger rotors. The wind speed is measured at several wind directions, depending on the shape of the building, allowing for a $360^{\circ}$ description of the flow conditions at varying wind angle.
- Roof-farm configuration: a combination of positions is detected on the roof where wind turbines are likely to be installed, and flow conditions are collected. The limit wind energy power density is calculated.

- Wind Turbine power characteristics: the roof-farm is equipped with suitable devices. Based on the installation height, a suitable diameter and rated power for the rotor is selected. The power curve together with the rated power is then modelled, registering cut-in and cut-out velocity. The power coefficient can be calculated and eventual discrepancies in the data would be corrected. The power curve could eventually be modified to take the effect of local flow features, such as wind shear of turbulence, into account.

- Climate data: the Weibull distribution for the ambient wind speed is retrieved based on the location of the high-rise buildings. Normally, such distributions are based on velocity bins and number of observations in terms of hours in a year. The distribution of the wind direction is also retrieved and essential to the calculation of the AEP.

For a wind turbine in operation, the power $P$ produced at a specific location can be found from the wind turbine power curve $P(u)$ and the probability distribution of the wind resource at that location $f(u)$ :

$$
P=\int_{u_{\text {in }}}^{u_{\text {out }}} P(u) f(u) d u(\mathrm{~W})
$$

where $u_{i n}$ and $u_{o u t}$ are respectively the cut-in and cutout wind speed for the wind turbine. Usually, equation (1) is applied to a limited range of time where the flow conditions are steady, e.g. $T=10 \mathrm{~min}$ or $1 \mathrm{hr}$. A way to estimate the performance of the roof farm is to consider a reference power:

$$
P_{r e f}=\frac{1}{2} \rho U^{3}\left(\frac{\mathrm{W}}{\mathrm{m}^{2}}\right)
$$

where $U$ can be defined based on the average wind speed at the installation location over the chosen range of time (i.e. the reference free-stream wind speed $U_{\text {ref }}$ for the high-rise building location). The performance of a wind turbine can also be estimated by calculating the ratio of power produced in a range of time $P$ normalised with the rated power $P_{r}$ of the wind turbine:

$$
C F=\frac{P}{P_{r}}
$$

$C F$ is normally calculated on annual base, binning the velocity into 10 -min equivalent windows. A realistic value for a small wind turbine is $C F \sim 0.3$, as from fullscale measurements. In this theoretical case, higher values are likely to be obtained in some specific configurations. In equation (1), the wind resource is introduced in the calculation of the total power $P$, using its probabilistic distribution $f(u)$. The Weibull distribution accurately describes the probabilistic behavior of atmospheric wind and it is used in meteorology: 


$$
f(u)=\frac{k}{c}\left(\frac{u}{c}\right)^{k-1} e^{-(u / c)^{k}}
$$

where $k$ is the shape parameter, which normally varies between 1.5-2.5 for atmospheric winds, and $c$ is the scale parameter, influenced by the mean of the distribution. Analogously to the normal distribution, $k$ and $c$ are related to each other through the mean wind speed $U=\bar{u}$ and the standard deviation $\sigma_{u}=\sqrt{\overline{u^{2}}}$ :

$$
U=c \Gamma\left(1+\frac{1}{k}\right) ; \sigma_{u}^{2}=c^{2}\left[\Gamma\left(1+\frac{2}{k}\right)-\Gamma^{2}\left(1+\frac{1}{k}\right)\right]
$$

where $\Gamma$ is the gamma function $\Gamma(x)=\int z^{x-1} e^{-z} d z$. For a given wind speed realization of duration $T$, the Weibull distribution parameters can be calculated and adapted to the free-stream wind speed, assuming that the ratio between local wind speed and free-stream wind speed is not affected by scale effects, i.e. $U / U_{\text {ref }}$ is constant. In this case, wind tunnel results can be generalized to calculate wind speed conditions at the installation site of wind turbines for a given free-stream wind speed. If the local flow features have similar characteristics with respect to the global flow features, the shape parameter $k$ is also bound to be constant, and therefore the scale parameter can be directly calculated:

$$
c=\frac{1}{\Gamma\left(1+\frac{1}{k}\right)} \frac{U}{U_{r e f}}
$$

The AEP for a given site can be calculated using equation (7) combined with equations (4) and (1), using the annual distribution for the mean free-stream wind speed $f\left(U_{\text {ref }}\right)$ :

$$
E=T \sum_{j} \int_{U_{r e f, 0}}^{U_{r e f, j}} P\left(U_{r e f}\right) f\left(U_{r e f}\right) d U_{r e f}
$$

where $T=8760 \mathrm{hr} /$ year, $U_{\text {ref } j \mathrm{j}}$ represents the free-stream velocity bins defining the ambient wind resource, $P\left(U_{\text {ref }}\right)$ is calculated using equation (1), and $f\left(U_{\text {ref }}\right)$ using equations (4) and (7) for the locations of interest above the roof.

\section{Wind Energy Resource}

\subsection{Wind Tunnel Experiments}

Extensive details on the experimental setup are given in $[6,14]$. The high-rise building model used in this study is a square prism, with sizes $H=0.4 \mathrm{~m}$ and $D=0.1333 \mathrm{~m}$, being equivalent to a $120 \mathrm{~m}$ high building, if referring to the wind tunnel scale of $1: 300$. The roof of the building is flat with sharp corners. The scale of the geometry is estimated according to the available atmospheric wind profile, reproducing a power-law with an exponent 0.2 and $U_{r e f}=U(H)=16 \mathrm{~m} / \mathrm{s}$ at $z=120 \mathrm{~m}$. The longitudinal turbulence intensity is $I_{u}(H) \sim 13 \%$, and the vertical one is $I_{w} \sim 11 \%$. Results at such wind speed are collected at $z=12 \mathrm{~m}$, using a miniature $\times$-wire hot-wire anemometer (HWA) measuring vertical and longitudinal wind component using a traverse system. The flow is measured over 7 positions above the roof, and 4 wind directions $\alpha=0^{\circ}, 15^{\circ}, 30^{\circ}$ and $45^{\circ}$. The symmetry of the building allows for interpolating and retrieving the velocity values over the whole roof surface and for other flow directions. Thus, a full directional study $0^{\circ}-360^{\circ}$ over 9 positions, namely 4 corners, 4 edges and the center of the roof, was possible. Adding up the height, this lead to a dataset of $9 \times 4 \times 1=36$ points.

\subsection{Wind turbine power curve}

The AEP of a wind farm is obviously influenced by the chosen wind turbines that compose it. However, the choice of a suitable small wind turbine is not trivial, as many devices are available on the market. The large majority of devices is composed by horizontal-axis wind turbines (HAWT), having a large variability of characteristics, such as size, number of blades, generators. Vertical-axis wind turbines (VAWT) are the second most common option.

The dataset for the implementation of wind turbines in this study is retrieved from [9], updating their reference to the Catalogue of European Manufacturers [15]. It is significant that many of the companies referred to in that catalogue have gone out of business. What is also noticeable is the large variability of rotor diameters and rated power for the dataset, respectively $D=0.5-14 \mathrm{~m}$ and $P_{r}=0.4-30 \mathrm{~kW}$. In fact, a commonly accepted definition for small wind turbine is yet to be agreed upon $[16,17]$.

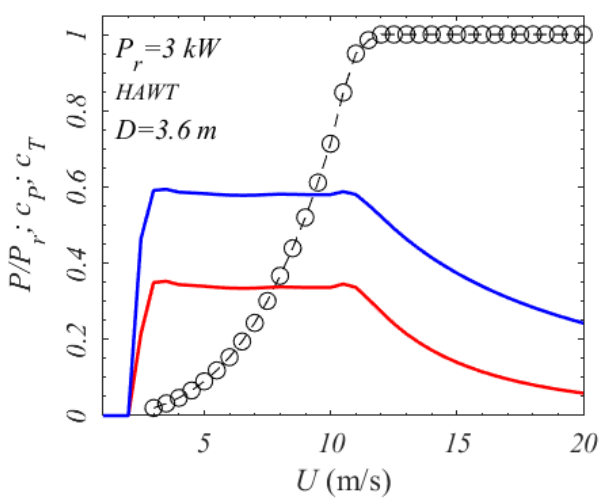

Figure 1. Power curve and Power coefficient of the HAWT used in this study. The rated power is also reported.

Table 1. Wind Turbine characteristics as implemented in this study.

\begin{tabular}{ccccccccc}
\hline \multirow{2}{*}{ Type } & $\begin{array}{c}P r \\
\mathrm{~kW}\end{array}$ & $\begin{array}{c}D \\
\mathrm{~m}\end{array}$ & $\begin{array}{c}A \\
\mathrm{~m}^{2}\end{array}$ & $\begin{array}{c}u_{\text {in }} \\
\mathrm{m} / \mathrm{s}\end{array}$ & $\begin{array}{c}u_{\text {out }} \\
\mathrm{m} / \mathrm{s}\end{array}$ & $\begin{array}{c}u_{R} \\
\mathrm{~m} / \mathrm{s}\end{array}$ & $\begin{array}{c}r p m \\
1 / \mathrm{min}\end{array}$ & $\begin{array}{c}Z_{\min } \\
\mathrm{m}\end{array}$ \\
\hline HAWT & 3.0 & 3.6 & 10.2 & 2.8 & $20^{*}$ & 12 & 550 & 3
\end{tabular}

In the present study, the power curve $P(u)$ of a HAWT suitable for installation in the built environment is retrieved from manufacturers data. The power coefficient is calculated in the range $u \in[0,20] \mathrm{m} / \mathrm{s}$ :

$$
c_{P}=\frac{P(u)}{\frac{1}{2} \rho u^{3}}
$$

where $\rho=1.25 \mathrm{~kg} / \mathrm{m}^{3}$ is the density of air. The cut-in and cut-out wind speeds $u_{\text {in }}$ and $u_{\text {out }}$ are also inputs in the 
dataset. It is significant to note that most small wind turbines do not have a defined cut-out wind speed. This is probably the result of early applications not producing energy due to the control system feathering the device when it detected $u>u_{\text {out }}$, often wrongfully due to the highly turbulent installation locations.

Figure 1 shows the normalized power curve and relative power coefficient of the HAWT chosen for this study. The HAWT is pitch-regulated, with a plateau after the rated velocity $P\left(u_{R}\right)=P_{r}=3 \mathrm{~kW}$, and can be installed at the minimum height of at $z=3 \mathrm{~m}$. The diameter of the rotor is $D=3.6 \mathrm{~m}$. Table 1 reports other wind turbine characteristics, which might be of interest.

\subsection{Roof-farm configurations}

As wind farms are set up considering the possible interaction among rotors, similarly roof-farm setups can be hypothesized by placing a number of wind turbines in various locations (see Figure 2). Single wind turbines can be placed on the corner, on the edge or at the center of the roof. Alternatively, multiple wind turbines can be placed with configurations s1-s5 exploiting the fact that corners or edges might be upwind or downwind. Finally, configurations $\mathrm{m} 1-\mathrm{m} 6$ take into consideration the use of a couple of wind turbines placed in areas of the flow which might be alternatively upwind and downwind, in an attempt to maximize performance.

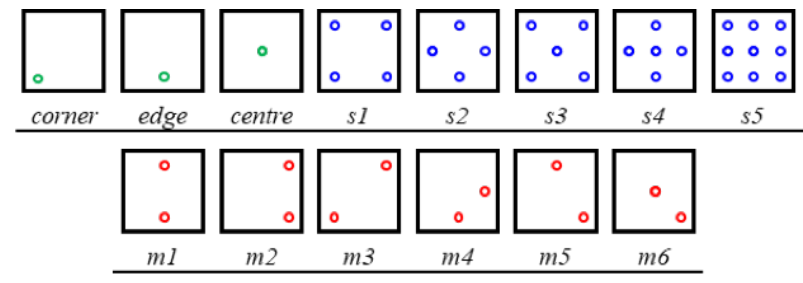

Figure 2 Hypothesized roof-farm setups, including single, small and medium wind turbines configurations.

\section{Results}

\subsection{Flow pattern}

The flow pattern above the roof of a high-rise building is complex, characterized by the presence of separation bubbles and cone vortices, which strongly vary with the wind direction. In order to install wind turbines and set up a roof-farm, the wind resource needs to be carefully characterized. In a previous study [14] the characterization of the flow is treated into details. However, in the present study the main findings are presented to interpret the conditions wind turbines are operating at in terms of wind speed, turbulence intensity and skewed flow.

Figure 3 shows the mean wind speed as recorded in the wind tunnel test, for four wind directions (columns) and at $z=12 \mathrm{~m}$. The center location of the roof is optimal for the positioning of wind turbines, as the highest wind speed are recorded with peaks of $U / U_{\text {re } ~} \sim 1.3$. At $\alpha=30^{\circ}$ and $45^{\circ}$, the wind speed is slightly smaller, with underperforming corners if compared to the roof center.
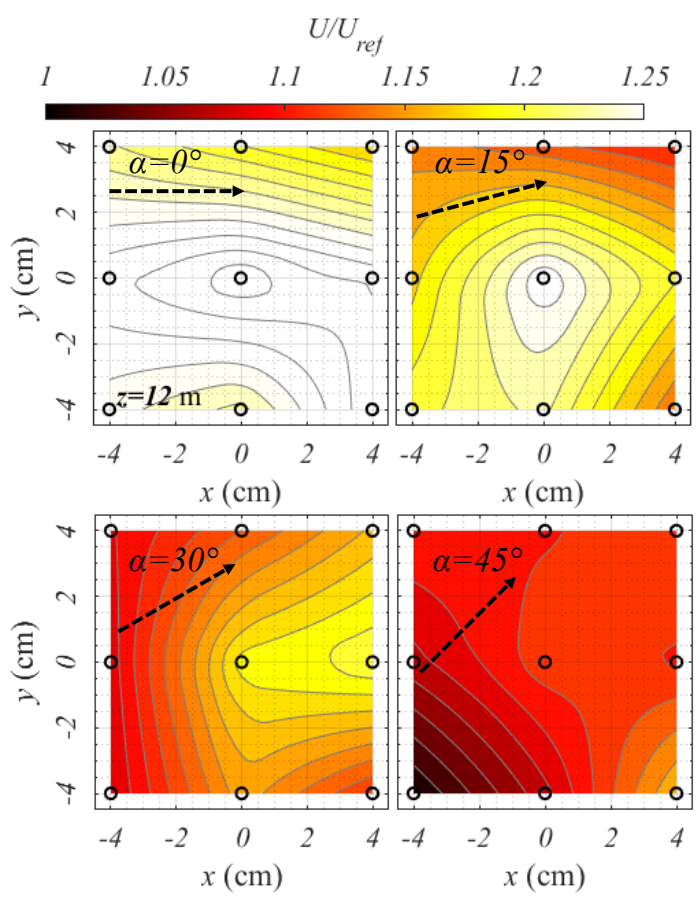

Figure 3 Mean Wind Speed at $\alpha=0^{\circ}, 15^{\circ}, 30^{\circ}$ and $45^{\circ}$ (see also dashed arrows) measured at $z=12 \mathrm{~m}$
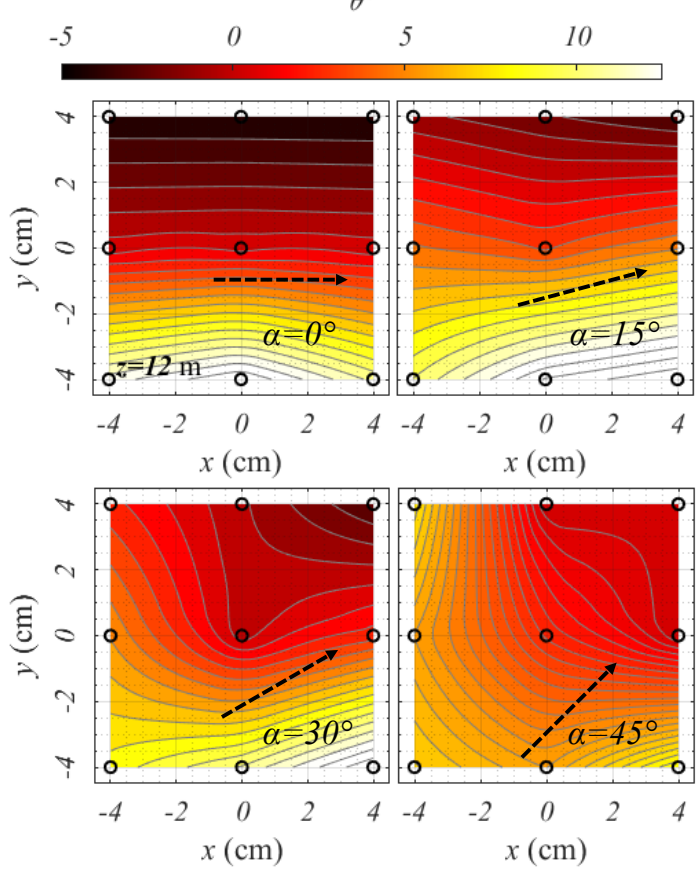

Figure 4. Skew angle $\theta^{\circ}$ at $\alpha=0^{\circ}, 15^{\circ}, 30^{\circ}$ and $45^{\circ}$ (see also dashed arrows) measured at $z=12 \mathrm{~m}$.

The particularly strong flow acceleration occurring at $\alpha=0^{\circ}$ and $15^{\circ}$ is due to the enhanced flow conditions found at this wind direction. Due to the higher Drag coefficient of the building, the obstacle imposed by the building causes the flow to accelerate above it, producing a beneficial configuration for the wind energy. The $z=3 \mathrm{~m}$ height alone is presented, given that above it the turbulence intensity is uniformly distributed with a value analogous to the inflow $I_{u} \sim 10-12 \%$. The wind direction also plays a major role in the skewed angle $\theta^{\circ}$ wind turbines operate at. The skew angle is defined as $\theta=\arctan (u / w)$.

Figure 4 shows that $\theta \sim 10^{\circ}$ at all wind directions close to the upwind edge, and then it recovers towards 
$\theta \sim 0-5^{\circ}$ on the downwind half of the building, with a nose-down condition at $\alpha=0^{\circ}$. This indicates that the funneling of the wake of the high-rise building is yet to take place, and above the roof there is a consistent skewed flow affecting the installation site of wind turbines. When installing medium sized rotors this could significantly affect performance, especially if HAWT are considered. On the contrary, VAWTs might be rather insensitive to skewed flow conditions [13]. This rather brief review of the flow conditions above the roof of high-rise buildings shows that the positioning of wind turbines is complicated, and much affected by the wind direction. If it is beneficial to raise the installation height, it is advisable to seek a height $z \sim 0.3 D$, where the beneficial effect of the accelerated flow is present but not affected by a significant increase in the turbulence intensity.

\subsection{Local Probability Distribution}

The main limitation of the previous section is that bulk statistics are used to calculate the wind energy density, without taking into account the probabilistic distribution or the response of wind turbines to such flow conditions. In this section, an approach that takes into account the probabilistic distribution based on equations (1) and (4) (Figure 2) for the response of wind turbines to flow conditions is used to calculate the energy output. The Weibull shape and scale parameters $c$ and $k$ are found for each position and then integrated with the power curves shown in Figure 1 to obtain the power generated by each configuration.

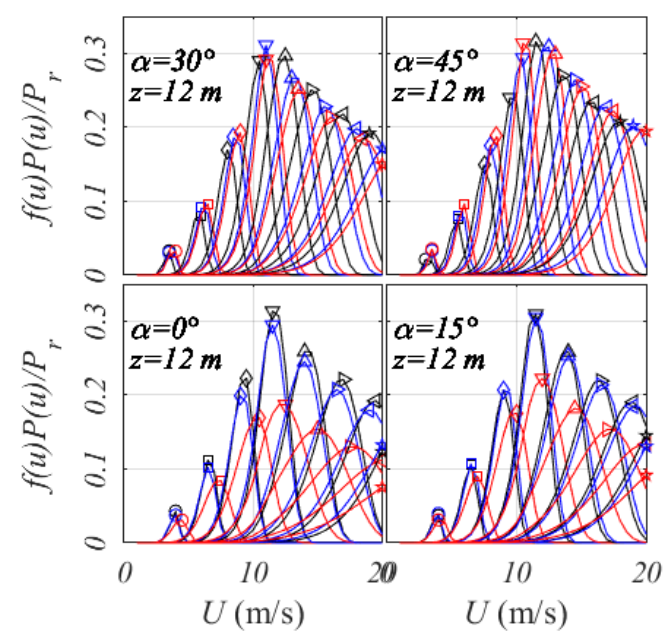

Figure 5. Integral for the calculation of power output as in equation (1).

As the reference velocity used in the wind tunnel is fixed at $U_{r e f}=16 \mathrm{~m} / \mathrm{s}$, the free-stream wind speed is assumed to be conveyed across the roof positions in the same way for different wind speeds $U_{r e f, i}$. It is hence possible to calculate the distribution at each position and the relevant integrand using equation (7). This is not a too far-fetched assumption, as the building has sharp edges. Therefore, a weak variability of its Drag and Lift coefficient is to be expected with the Reynolds number. The integral $f(u) P(u) / P_{r}$ is shown in Figure 5. Black lines represent corner position, blue lines refer to the edges and red lines to the center of the roof top. Each different position, depending on $\mathrm{c}$ and $\mathrm{k}$, shows a different distribution for a given $U_{\text {ref }, i}$. Given that the total energy depends on the AEP calculated integrating $f(u) P(u) / P_{r}$, the undisturbed freestream atmospheric wind is included in terms of how many hours each distribution has been integrated for. Figure 5 shows the result of this approach for $U_{\text {ref }}=3(0), 6(\square), 9(\diamond), 12$ $(\nabla), 15(\triangle), 18(\triangleright), 21(\triangleleft)$ and $24 \mathrm{~m} / \mathrm{s}(\lesssim)$. At each reference velocity the integral varies due to the power curve of the chosen wind turbine. Figure 5 demonstrates that at $\alpha=45^{\circ}$ a significantly higher power can be harvested than at $\alpha=0^{\circ}$, which shows limited performance especially at the center positions. In fact, looking at the peak values the performance at $\alpha=0^{\circ}$ at the corner and the edge is analogous to that depicted at $\alpha=45^{\circ}$ and a similar amount of power can be extracted. This is consistent with results from Figure 3. By further integrating for each velocity $U$, the total power extracted by the system can be calculated using equation (8).

\subsection{Annual Energy Distribution in Firenze (IT)}

In order to determine the performance of a wind farm, its performance over a yearly basis needs to be calculated. To that end, the annual wind speed distribution is needed to calculate the number of hours a wind speed is to be expected on an installation site. Figure 6 shows the annual Weibull distribution for the wind speed in the city of Firenze (IT). This location is interesting, as its vicinity to the Apennine mountains causes the main yearly wind to have a specific northerly direction (in Figure 6 the direction $0^{\circ}$ refers to wind blowing from $N$ ). The Weibull distribution has a shape parameter of 1.2, with a mean wind speed of $U_{\text {ref }}=3.93$ $\mathrm{m} / \mathrm{s}$, yielding a shape factor of 4.15 .
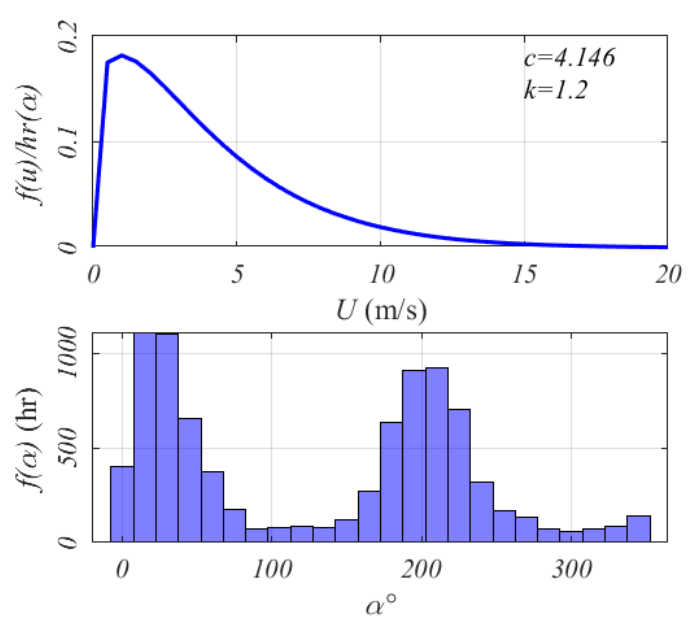

Figure 6. Climate characteristics in Firenze (IT).

In Northern Europe, Weibull distribution have generally a higher shape factor $k \sim 2$. From the Weibull distribution of the atmospheric weather it is possible to integrate the power produced at the direction bin $U_{r e f, i}$ times the number of hours of that bin, assuming that for each bin the same Weibull distribution holds. That is a strong hypothesis, since usually each bin has a different Weibull distribution (i.e. depending on the terrain roughness, orography and other parameters). 
The total number of hours of the distribution is to be calculated from the probability distribution for the wind direction. If we consider a uniform wind rose, meaning each $15^{\circ}$ sector contains $8760 / 24=365 \mathrm{hr}$, then the wind speed Weibull distribution can be applied at each sector multiplying the probability times the hours in the sector. Results are then conveyed to the building thanks to wind speed measurements showed in the previous section and the produced power for that bin at that sector is hence calculated. Figure 7 shows the Annual Energy (AE) output for the roof farm against each wind direction and for each roof-farm configuration. As expected, configuration s5 (the eight one, colored with light purple) produces the highest amount of energy, being composed by nine wind turbines. So do configurations s3-s4 (sixth and seventh configuration) and s1-s2 (fourth and fifth). It is worth underlining that in a year, a rooffarm composed by one wind turbine is able to produce $\sim 1.3$ MWh of energy.
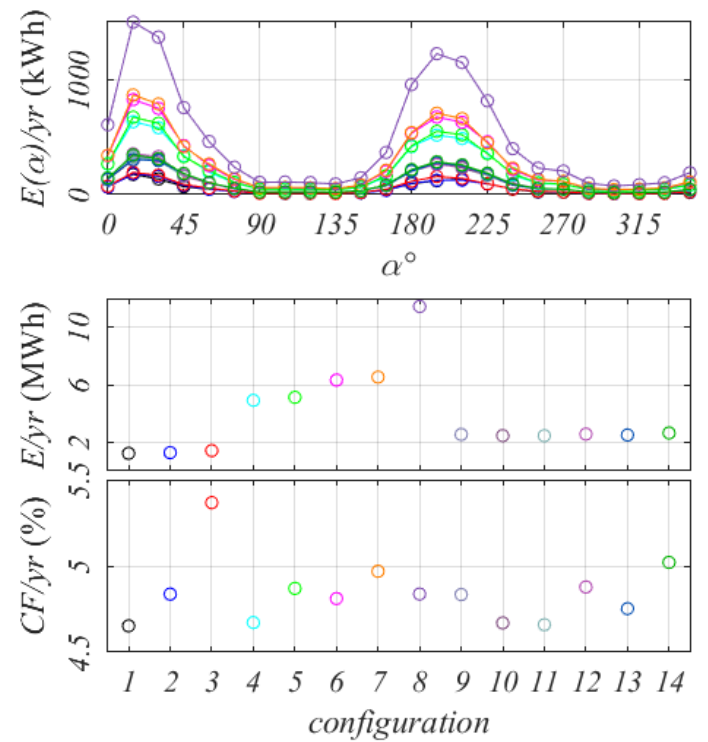

Figure 7. AE produced for each wind direction (above); Total AE produced in a year (centre); $\mathrm{CF}$ of the roof-farm at $z=12 \mathrm{~m}$ (below); configurations refer to Figure 2 as follows: 1-3 corner-edge-center; 4-8 s1-s5; 9-14 m1-m6)

The Capacity Factor (CF) allows for inferring how well the wind turbines are exploited in terms of the rated power. CF is analogous for all configurations, with $\mathrm{CF}>5 \%$ only for the center location, and configurations s4 and m6, which include the center location amongst the positions wind turbines are installed at. Corners in general underperform with respect to edges, as shown by configurations 2, 5 and 7 in Figure 7 . This is also verified looking at configurations 10,11 and 13 , which feature wind turbines in the corners. The low $\mathrm{CF}$ value can be interpreted with the fact that the roof-farm is rather sensitive to the wind direction for this shape of the building.

\section{Conclusions}

Based on the results showed in the present paper, it is possible to evaluate that up to $6 \mathrm{MWh}$ can be produced with a roof-farm using the most promising configuration. Considering that for a typical building in central Italy the yearly consumption of electricity is $\sim 100 \mathrm{kWh} / \mathrm{m}^{2}$ and assuming that the usable area of a high-rise building is $\sim 30 \times 30=900 \mathrm{~m}^{2}$ for 40 floors, the yearly consumption of electricity amounts to $\sim 3600$ MWh. Then, a roof farm set up could eventually cover up to $0.2 \%$ of the energy request. That value may vary greatly depending on the typology of the wind turbine installed, the impact of mutual interaction between the wind turbines and by optimizing the roof-farm against the flow pattern, the shape of the building and main wind directions. Therefore, the procedure showed in the present paper could be used to study several building shapes, also considering the support of high-fidelity numerical methods to better define the local characteristics of the flow field.

\section{References}

1. Hamza, N., Trends and Challenges for Wind Energy Harvesting: Workshop. Coimbra, Portugal (2015)

2. Micallef D., van Bussel G., Energies 11, 2204 (2018)

3. Stathopoulos T., Alrawashdeh H., Al-Quraan A., Blocken B., Dilimulati A., Paraschivoiu M., Pilay P., J. Wind Eng. Ind. Aerodyn. 179, 146-157 (2018)

4. Evans S.P., KC A., Bradney D.R., Urmee T.P., Whale J., Clausen P.D., Renew. Energy Environ. Sustain. 2, 31 (2017)

5. Toja-Silva F., Peralta C., Lopez-Garcia O., Navarro J., Cruz I., J. Wind Eng. Ind. Aerodyn. 142 (2015)

6. Šarkić Glumac A., Hemida H., Höffer R., J. Wind Eng. Ind. Aerodyn. 175, 32-42 (2018)

7. Hemida H., Šarkic A., Höffer R., 14th International Conference on Wind Engineering. Porto Alegre, Brazil (2015)

8. Vita G., Šarkić-Glumac A., Hemida H., Salvadori S., Baniotopoulos C., Energies, under review (2020)

9. Drew D.R., Barlow J.F., Cockerill T.T., J. Wind Eng. Ind. Aerodyn. 115, 104-111 (2013)

10. Rezaeiha A., Montazeri H., Blocken B., Energy Convers. Manag. 214, 112770 (2020)

11. Rezaeiha A., Montazeri H., Blocken, B., Energy 165, 1129-1148 (2018)

12. Ledo L., Kosasih P.B., Cooper P., Renew. Energy 36, 1379-1391 (2011)

13. Balduzzi F., Bianchini A., Ferrari L., Renew. Energy 45, 163-174 (2012)

14. Vita G., Hemida H., Andrianne T., Baniotopoulos C., J. Wind Eng. Ind. Aerodyn., accepted (2020)

15. WINEUR, Catalogue of European Urban Wind Turbine Manufacturers (2007)

16. Gsänger S., Pitteloud J.-D., 2015. Small Wind World Report (2015)

17. Tummala A., Velamati R.K., Sinha D.K., Indraja V., Krishna V.H., Renew. Sustain. Energy Rev. 56, 13511371 (2016)

18. Toja-Silva F., Lopez-Garcia O., Peralta C., Navarro J., Cruz I., Appl. Energy 164, 769-794 (2016) 